\title{
Estrategia educativa para incrementar el cumplimiento del régimen antituberculoso en Chiapas, México
}

\author{
Guadalupe del Carmen Álvarez Gordillo, ${ }^{1}$ Julio Félix Álvarez Gordillo ${ }^{2}$ \\ y José Eugenio Dorantes Jiménez ${ }^{3}$
} RESUMEN Objetivo. Aplicar un plan de capacitación para médicos y pacientes y evaluar su eficacia en
términos del cumplimiento por parte de los pacientes del tratamiento contra la tuberculosis pulmonar en la región fronteriza de Chiapas, México.

Métodos. Se realizó un estudio de intervención controlado en pacientes mayores de 15 años con tuberculosis pulmonar diagnosticada por baciloscopia entre el 1 de febrero de 2001 y el 31 de enero de 2002 en unidades de salud seleccionadas al azar en la región fronteriza de Chiapas, México. La muestra estuvo formada por pacientes que acudieron por demanda de consulta en ese período a 23 y 25 unidades de salud para cada uno de los grupos en estudio: intervención y control, respectivamente. En el grupo de intervención se aplicó un plan de capacitación para el personal de salud en el que se abordaron los aspectos sociales, culturales y económicos de la tuberculosis; las bases teóricas y prácticas del diagnóstico y el tratamiento de la enfermedad y la formación de grupos de autoayuda. También se formaron grupos de autoayuda para todos los pacientes que acudieron a las 23 unidades del grupo de intervención. A todos los pacientes se les administró un régimen terapéutico abreviado con isoniacida, rifampicina, pirazinamida y etambutol durante 25 semanas, hasta completar 105 dosis. El seguimiento de los pacientes se extendió hasta el mes de diciembre de 2003. Los grupos de intervención y control se compararon mediante la prueba de la ji al cuadrado y se empleó la prueba de la t de Student para comparar las medias. Se calculó el riesgo relativo (RR) de no cumplir y sus intervalos de confianza de 95\% (IC95\%).

Resultados. En la investigación participaron 87 pacientes: 44 en el grupo sometido a intervención y 43 en el grupo de control. El cumplimiento del tratamiento fue significativamente mayor en el grupo que recibió la intervención que en el grupo que sirvió de control (97,7\% frente a 81,4\%, respectivamente; $R R=1,20 ;$ IC95\%: 1,03 a 1,39; P =0,015).

Se observó que los médicos de la región fronteriza de Chiapas enfocan su actividad hacia la medicina curativa, más que a la preventiva y a la comprensión de las causas sociales de las enfermedades.

Conclusiones. Mediante las actividades educativas de la intervención se logró aumentar la proporción de pacientes que cumplieron el tratamiento. Los servicios de salud pueden mejorar el control de la tuberculosis en Chiapas con los recursos de los que disponen actualmente. Se debe transmitir a los médicos una visión integral de los problemas de salud en Chiapas y fomentar una mejor relación entre médico y paciente. También se debe procurar que los servicios de salud abran espacios educativos y de participación comunitaria que permitan enfrentar de manera global los problemas de salud pública.

Palabras clave Tuberculosis, cooperación del paciente, grupos de autoayuda, relaciones profesionalpaciente.

1 Colegio de la Frontera Sur, Chiapas, México. La correspondencia debe ser enviada a Guadalupe Álvarez Gordillo, Colegio de la Frontera Sur, Ca- rretera Panamericana y Periférico Sur s/n, San Cristóbal de Las Casas, Chiapas, México. Correo electrónico: galvarez@sclc.ecosur.mx
2 Secretaría de Educación Pública, Chiapas, México. 3 Instituto de Salud del Estado de Chiapas, Jurisdicción Sanitaria III, Chiapas, México. 
La tuberculosis ha resurgido como un problema de salud pública de alcance mundial debido principalmente a la aparición de cepas de Mycobacterium tuberculosis resistentes a los fármacos y a que la tuberculosis se ha notificado como la causa de muerte en la tercera parte de los casos de sida (1-4). La resistencia a los medicamentos obedece a errores humanos, en particular a la falta de cumplimiento del tratamiento antituberculoso prescrito, la cual hace que proliferen cepas bacterianas resistentes que reducen las posibilidades de curación.

En México, la incidencia de la tuberculosis pulmonar ha mantenido un incremento constante en los últimos años y pasó de 14,7 casos por 100000 habitantes en 1990 a 15,6 por 100000 habitantes en el año 2000 (5). Ese año, Chiapas ocupó el primer lugar en defunciones por tuberculosis entre todos los estados de ese país, con una tasa anual de mortalidad por esta enfermedad de 14,7 por 100000 habitantes en hombres y de 9,5 por 100 000 habitantes en mujeres (6). Ese mismo año, la filial del Instituto de Salud del Estado de Chiapas en la región fronteriza notificó 121 casos de tuberculosis, lo que equivale a una tasa de mortalidad de 26,7 por 100000 habitantes. En el período de 1989 a 1999, el tratamiento se mostró eficaz en un promedio de $75 \%$ de los casos (porcentaje de pacientes curados en relación con los que iniciaron el tratamiento) (7).

La población de Chiapas es muy diversa en términos lingüísticos $\mathrm{y}$ culturales y está muy dispersa: $40 \%$ de los habitantes viven en localidades rurales y $17,7 \%$ pertenecen a grupos étnicos autóctonos. En general, las condiciones de vida son malas y se caracterizan por viviendas precarias, hacinamiento y carencia de saneamiento ambiental básico. La desnutrición afecta a $41 \%$ de la población menor de 5 años y el índice de analfabetismo es de $26 \%$. Por todo ello, no es de extrañar que la prevalencia de tuberculosis en la región sea tan alta (8). Además, la percepción que se tiene de la enfermedad en la zona influye negativamente en la utilización de los servicios de salud y en la curación de los enfermos $(9,10)$.

La forma en que se percibe la tuberculosis en las diferentes regiones de Chiapas está ligada a la experiencia personal y a la influencia de las relaciones sociales que rodean a cada persona. Por lo tanto, las decisiones que toman los enfermos en cuanto a dónde y cuándo buscar ayuda o a la elección de una unidad de salud en especial dependen del conocimiento que tienen de su enfermedad y de la experiencia acumulada en el seno de la familia. Otro elemento importante en este sentido es la red de apoyo social e institucional, ya que esta es capaz de influir sobre los valores de los enfermos y sus necesidades de salud $(11,12)$.

A pesar de que ya se ha comprobado la eficacia de la estrategia del tratamiento acortado estrictamente supervisado (14), es necesario evaluar otras estrategias complementarias que abarquen tanto a los enfermos como a los servicios de salud para lograr modificar la conducta, ya que esto puede tener un mayor impacto a largo plazo $(15,16)$.

El objetivo de este estudio fue aplicar un plan de capacitación para médicos y pacientes y evaluar su eficacia en términos del cumplimiento por parte de los pacientes del tratamiento contra la tuberculosis pulmonar en la región fronteriza de Chiapas, México.

\section{MATERIALES Y MÉTODOS}

\section{Diseño del estudio}

Se realizó un estudio de intervención controlado en pacientes mayores de 15 años con tuberculosis pulmonar diagnosticada por baciloscopia entre el 1 de febrero de 2001 y el 31 de enero de 2002 en unidades de salud seleccionadas al azar afiliadas a la Secretaría de Salud (SSA), el Instituto Mexicano del Seguro Social (IMSS) y el Instituto de Servicios y Seguridad Social para los Trabajadores del Estado (ISSSTE), todos de la región fronteriza de Chiapas, México. El seguimiento de los pacientes se extendió hasta el mes de diciembre de 2003.

\section{Selección de las unidades de salud participantes}

La Jurisdicción Sanitaria III del estado mexicano de Chiapas, ubicada a lo largo de la frontera con Guatemala, está conformada por ocho municipios con una población total de 373527 habitantes en 1999 (8). Estos son Comitán, Chicomuselo, Frontera Comalapa, La Independencia, Las Margaritas, Socoltenango, La Trinitaria y Tzimol. Todos los casos de tuberculosis se informan a la SSA, que cuenta con 24 unidades médicas de primer nivel, un hospital y 10 unidades médicas móviles; al IMSS, que cuenta con 92 unidades del régimen solidaridad (atención a la población sin seguro médico formal) y 2 de régimen ordinario (atención a la población con seguro médico pagado por alguna empresa); y al ISSSTE, que tiene 3 unidades médicas familiares o de primer nivel de atención (17). Sin embargo, no todas las unidades notifican casos de tuberculosis de forma continua.

Se conformaron dos grupos de unidades: una de intervención y una de control. Con vistas a tener un número similar de casos en ambos grupos, las unidades de salud que notificaron algún caso de tuberculosis durante el año 2000 se identificaron con códigos que se pusieron en una urna de la que luego se extrajo al azar el número de unidades que participarían en la estrategia educativa, buscando completar la mitad de los casos de tuberculosis diagnosticados y atendidos ese año. Los pacientes vistos en el resto de las unidades, que atendieron a un número similar de pacientes, integraron el grupo de control. En el grupo de intervención (23 unidades), el personal sanitario recibió capacitación sobre los aspectos sociales, culturales y económicos de la tuberculosis, las bases teóricas y prácticas del diagnóstico y el tratamiento de la enfermedad, y la formación de grupos de autoayuda; por su parte, los pacientes participaron en reuniones de autoayuda. En el 
grupo de control (25 unidades) las actividades clínicas de seguimiento del tratamiento continuaron realizándose según las normas establecidas por el Programa Estatal de Tuberculosis, sin actividades de capacitación ni autoayuda.

\section{Descripción de la intervención}

Capacitación del personal sanitario. Durante el tiempo que duró la captación de casos se llevaron a cabo tres talleres multidisciplinarios de dos días cada uno para el personal médico y de enfermería y para los trabajadores sociales de las unidades de salud del grupo de intervención. Los temas de discusión se definieron a partir de entrevistas previas realizadas a 13 médicos de la SSA y el IMSS acerca de $\mathrm{su}$ formación profesional y su experiencia en lo referente a la relación entre el médico y el paciente. Estos profesionales fueron seleccionados intencionalmente con diferentes años de experiencia entre los que generalmente atendían a pacientes con tuberculosis y aceptaron participar en la investigación. Los temas fueron multidisciplinarios y abarcaron los aspectos sociales, culturales y económicos de la tuberculosis; las bases teóricas y prácticas de su diagnóstico y tratamiento; la formación de grupos de autoayuda; y la descripción y análisis de las diferentes actividades y lineamientos del Programa de Prevención y Control de la Tuberculosis, tanto nacional como estadual y regional. Los talleres fueron coordinados por la investigadora médica responsable del estudio y en ellos participaron como invitados la responsable del programa de tuberculosis jurisdiccional, el epidemiólogo jurisdiccional, un sociólogo y una enfermera, todos con experiencia en el tema. El objetivo fue brindar los elementos necesarios para entender la complejidad de la enfermedad, el contexto y el cumplimiento del tratamiento. Se insistió acerca de la necesidad de mejorar la relación entre el médico y el paciente y de lograr una mayor comunicación entre el personal administrativo (encargado de dar las citas para las consultas, realizar los estudios socioeconómicos para la exención de las cuotas y programar las visitas domiciliarias en caso necesario) y el personal de enfermería y de laboratorio.

Grupos de autoayuda para pacientes con tuberculosis. Se formaron cuatro grupos de autoayuda de 2 a 10 enfermos cada uno. Las reuniones se realizaron mensualmente bajo la coordinación de los médicos en las mismas unidades de salud donde los pacientes recibían su tratamiento, aunque cada grupo eligió libremente el número de sesiones y los temas de discusión, según sus necesidades de información y sus dudas. En tres grupos se realizaron cinco reuniones y en uno se realizaron seis. En las reuniones se discutió la percepción de la población acerca de la tuberculosis y se trataron temas relacionados con la atención médica, la alimentación, los mecanismos de transmisión de la enfermedad y su diagnóstico, así como con el tratamiento y su cumplimiento por parte de los pacientes. Se prepararon materiales audiovisuales para la presentación clara y sencilla de los diferentes temas. En las unidades de salud que no diagnosticaron tuberculosis en más de un paciente por año no se pudieron conformar grupos de autoayuda.

\section{Criterios de inclusión y exclusión}

En el estudio participaron todos los pacientes mayores de 15 años en quienes se diagnosticó tuberculosis pulmonar mediante baciloscopia de esputo entre el 1 de febrero de 2001 y el 31 de enero de 2002 y que aceptaron someterse a tratamiento. A los pacientes se les aplicó un esquema terapéutico abreviado con isoniacida, rifampicina, pirazinamida y etambutol durante 25 semanas hasta completar 105 dosis; las primeras 60 dosis se aplicaron diariamente de lunes a sábado durante 10 semanas (fase intensiva) y las 45 dosis restantes, sin etambutol, tres veces por semana en días alternos durante 15 semanas (fase de sostén) (18). Durante el tratamiento y seguimiento de los casos se obtuvo información de los expedientes clínicos, de las tarjetas de control de tratamiento de los pacientes y de los propios pacientes, en el momento de asistir a las reuniones de los grupos de autoayuda, acerca de la duración y evolución de la enfermedad, las fechas de diagnóstico y tratamiento y el resultado de las pruebas de baciloscopia realizadas mensualmente. Esta información se recolectó en un formato diseñado con ese fin y luego se introdujo en una base de datos.

Se excluyó a los pacientes con tuberculosis extrapulmonar y a los que tenían antecedentes de resistencia a los antibióticos.

Se consideró que el tratamiento se había cumplido si el paciente había tomado, al final del estudio, 75\% o más de las dosis prescritas. En cambio, el tratamiento se consideró abandonado si el paciente lo había interrumpido durante 30 días o más. Se consideraron curados los casos de tuberculosis que al terminar el tratamiento primario no presentaban signos clínicos y tenían resultados de baciloscopia negativos en dos muestras mensuales sucesivas, así como aquellos casos en los que al término del tratamiento no presentaban signos clínicos ni expectoración.

\section{Análisis estadístico}

Se utilizó el programa Epi Info versión 6.04 (19) para el análisis de los datos. Los grupos de intervención y control fueron comparados mediante la prueba de la ji al cuadrado y se empleó la prueba de la $t$ de Student para comparar las medias. Se calculó el riesgo relativo (RR) de no cumplir y sus intervalos de confianza de $95 \%$ (IC95\%). Para analizar la diferencia estadística entre las proporciones de pacientes que cumplieron el tratamiento en ambos grupos se $u$ tilizó la prueba de la $Z$ para diferencias entre dos proporciones de población, con intervalos de confianza de $95 \%$ (20). El nivel de significación fue de $95 \%$ 


\section{RESULTADOS}

En la jurisdicción sanitaria se registraron 126 pacientes con tuberculosis durante el período de estudio. De ellos, 116 casos eran nuevos y 10 $(7,9 \%)$ de reingreso; se registraron 8 defunciones $(6,3 \%)$ a causa de la enfermedad. En 92 pacientes se llegó al diagnóstico de tuberculosis pulmonar por baciloscopia, de ellos uno falleció y 4 tenían antecedentes de tratamiento previo, en 2 de ellos se determinó que la tuberculosis era resistente a múltiples fármacos.

\section{Características de los pacientes estudiados}

Del total de 92 casos de tuberculosis pulmonar registrados, 87 pacientes cumplieron con los criterios de inclusión; 44 correspondieron al grupo sometido a intervención y 43 al grupo de control. No se observaron diferencias significativas entre los pacientes del grupo de estudio y del grupo de control en cuanto a edad ( $P=$ $0,989)$ ni sexo $(P=0,241)$ (cuadro 1$)$. El mayor número de pacientes provenía de los municipios Frontera Comalapa, Las Margaritas y Comitán, tanto en el grupo de intervención $(75,9 \%)$ como en el grupo de control (76.7\%). Los dos últimos municipios tenían unidades de salud incluidas en el grupo de intervención.

En la discusión generada en los grupos de autoayuda se reiteraron algunos elementos relacionados con la falta de cumplimiento del tratamiento que se habían detectado en estudios previos $(11,12)$. En este sentido, las dificultades encontradas con mayor frecuencia estaban relacionadas con un deficiente vínculo entre el médico y el paciente y el estigma que perciben en la enfermedad la mayoría de los pacientes, entre los cuales se detectaron conductas que los llevaban a ocultar su enfermedad y aislarse. Como ejemplo de ese tipo de actitud, seis pacientes manifestaron que a pesar de tener una unidad de salud cerca de su comunidad preferían viajar hasta 5 horas para recibir el tratamiento, a pesar de que ello

CUADRO 1. Características de los pacientes que participaron en el estudio. Chiapas, México, 2001-2002

\begin{tabular}{|c|c|c|c|c|c|}
\hline \multirow[b]{3}{*}{ Variable } & \multicolumn{4}{|c|}{ Grupo } & \multirow[b]{3}{*}{ Total } \\
\hline & \multicolumn{2}{|c|}{ Intervención } & \multicolumn{2}{|c|}{ Control } & \\
\hline & No. & $\%$ & No. & $\%$ & \\
\hline \multicolumn{6}{|l|}{ Sexo } \\
\hline Masculino & 25 & $(56,8)$ & 19 & $(43,2)$ & 44 \\
\hline Femenino & 19 & $(44,2)$ & 24 & $(55,8)$ & 43 \\
\hline \multicolumn{6}{|l|}{ Edad } \\
\hline 15-29 años & 19 & $(55,8)$ & 15 & $(44,2)$ & 34 \\
\hline 30-49 años & 9 & $(43,8)$ & 12 & $(56,2)$ & 21 \\
\hline 50-69 años & 11 & $(50)$ & 11 & (50) & 22 \\
\hline 70-89 años & 5 & $(50)$ & 5 & (50) & 10 \\
\hline \multicolumn{6}{|l|}{ Ocupación: } \\
\hline Agricultor & 19 & $(55,9)$ & 15 & $(44,1)$ & 34 \\
\hline Oficios del hogar & 14 & $(37,8)$ & 23 & $(62,2)$ & 37 \\
\hline Estudiante & 4 & $(80,0)$ & 1 & $(20,0)$ & 5 \\
\hline Albañil & 2 & $(100)$ & 0 & & 2 \\
\hline Otra & 3 & $(60)$ & 2 & $(40)$ & 5 \\
\hline Desempleado & 2 & (50) & 2 & (50) & 4 \\
\hline
\end{tabular}

implicaba mayores gastos de traslado. Estos pacientes no abandonaron el tratamiento cuando comenzaron a participar en las actividades educativas programadas para el grupo de intervención a pesar de que la carestía del transporte ha sido identificada como un factor que lleva a abandonar el tratamiento.

\section{Cumplimiento del tratamiento}

Según el Programa Jurisdiccional de Tuberculosis de la Región Fronteriza, el porcentaje de pacientes con tuberculosis pulmonar en la frontera del estado de Chiapas que cumplió su tratamiento antituberculoso durante el año 2000 fue de 80 (13). Mediante las actividades educativas emprendidas se logró que el cumplimiento aumentara en el grupo de intervención a $97,7 \%$, mientras que en el grupo de control solo fue de $81,4 \%(R R=1,20$; IC95\%: 1,03 a 1,39; $P=0,015$ ) (cuadro 2). La diferencia entre la proporción de pacientes que cumplieron el tratamiento en el grupo de intervención y en el grupo de control fue estadísticamente significativa $(Z=$ 2,85; IC95\%: 0,081-0,245; $P<0,05$ ).

En el grupo sometido a la intervención, un paciente abandonó el tratamiento después de haber cumplido $57,1 \%$ del tratamiento previsto (60 dosis), mientras que en el grupo de control, dos pacientes abandonaron el tratamiento después de 68 dosis $(64,8 \%$ del tratamiento) y

CUADRO 2. Medida en que cumplieron el tratamiento contra la tuberculosis los pacientes estudiados. Chiapas, México, 2001-2002

\begin{tabular}{|c|c|c|c|c|}
\hline \multirow[b]{2}{*}{ Grupo } & \multicolumn{2}{|c|}{ Cumplimiento $^{a}$} & \multicolumn{2}{|c|}{ Incumplimiento } \\
\hline & No. & $\%$ & No. & $\%$ \\
\hline De intervención & 43 & $97,7^{b}$ & 1 & 2,3 \\
\hline De control & 35 & $81,4^{b}$ & 8 & 18,6 \\
\hline Total & 78 & 89,6 & 9 & 10,4 \\
\hline
\end{tabular}


12 dosis (11,4\% del tratamiento), respectivamente. En este grupo se registraron cinco fracasos terapéuticos, todos en pacientes que interrumpieron el tratamiento por períodos menores de 30 días o que no completaron por lo menos $75 \%$ del tratamiento (cuadro 3 ). Los resultados del laboratorio demostraron que dos de ellos tenían tuberculosis resistente a varios antibióticos, sin haber declarado recibir tratamientos previos. Un paciente se trasladó a otra unidad, donde no pudo ser localizado para continuar el seguimiento.

Los $43(97,7 \%)$ pacientes del grupo de intervención y los $35(81,4 \%)$ del grupo de control que se clasificaron como curados y que cumplieron el tratamiento, según el criterio indicado anteriormente, tomaron $100 \%$ de las dosis, con excepción de un paciente del grupo de control que tomo $94,3 \%$.

\section{Talleres para el personal sanitario}

Durante el análisis de las entrevistas previas y los talleres se encontró que los médicos de la región fronteriza enfocaban su actividad hacia la medicina curativa, más que hacia la preventiva o hacia la comprensión de las causas sociales de las enfermedades. Hacían hincapié en el diagnóstico, la dieta y el tratamiento, así como en la exploración y evolución clínica. Esto se explica en parte por la presión administrativa de realizar hasta 30 consultas por día, llenar los formularios de diagnóstico y notificación, y la visión institucional que relega a un segundo plano el contexto cultural, social y afectivo en que se desenvuelven los pacientes.

Los profesionales manifestaron que a pesar de haber cursado asignaturas que abordaban el estudio de la comunidad y el trato del paciente, en la práctica profesional se presentan dificultades. Solo la experiencia enseña la manera de conducir la relación entre el médico y el paciente en los diferentes contextos socioculturales.

Los investigadores observaron entre los pacientes una actitud colaboradora y de comprensión de la utilidad de participar en los grupos de autoayuda.

CUADRO 3. Resultados clínicos observados en los pacientes estudiados. Chiapas, México, 2001-2002

\begin{tabular}{lccc}
\hline \multicolumn{1}{c}{ Clasificación final } & $\begin{array}{c}\text { Grupo de } \\
\text { intervención }\end{array}$ & $\begin{array}{c}\text { Grupo de } \\
\text { control }\end{array}$ & Total \\
\hline Curado $^{\text {a }}$ & 43 & 35 & 78 \\
$\quad$ Curado, con baciloscopia negativa & 40 & 34 & 74 \\
$\quad$ Curado, sin baciloscopia & 3 & 1 & 4 \\
Fracaso & 0 & 5 & 5 \\
Abandono & 1 & 2 & 3 \\
Traslado & 0 & 1 & 1 \\
$\quad$ Total & 44 & 43 & 87 \\
\hline
\end{tabular}

a Se consideraron curados, los casos de tuberculosis que al terminar el tratamiento primario no presentaban signos clínicos y los resultados de la baciloscopia fueron negativos en dos muestras mensuales sucesivas, así como aquellos casos en los que al término del tratamiento no presentaban signos clínicos ni expectoración.

\section{DISCUSIÓN}

Este estudio es el primero que evalúa los efectos de una estrategia educativa en el cumplimiento del tratamiento contra la tuberculosis en Chiapas, México. Se comprobó la necesidad de que los programas de prevención y control de enfermedades como la tuberculosis tomen en cuenta los intereses y la participación de los enfermos para lograr mejores resultados en el tratamiento.

La incorporación del personal de salud a talleres de capacitación multidisciplinarios permitió que se conocieran y comprendieran mejor los beneficios del cumplimiento estricto del tratamiento contra la tuberculosis. Además, los resultados obtenidos previos a la realización de los talleres reflejaron deficiencias en la formación profesional del personal sanitario, específicamente en lo concerniente al conocimiento del contexto social de las diferentes regiones de Chiapas y de las condiciones de vida de los pacientes. También se observó la falta de una visión integral del tratamiento como vía de lograr la curación de la enfermedad.

El tratamiento acortado estrictamente supervisado ha demostrado su eficacia en varias regiones del mundo (21-23). Sin embargo, de acuerdo con una revisión realizada por Volmink y colaboradores, muchos de esos estudios han sido publicados precisamente porque fueron exitosos o contaban con la influencia de coordinadores carismáticos. Algunas de estas publicaciones reflejan claramente el entusiasmo de sus realizadores, aunque este efecto sea difícil de cuantificar. Además, muchos de esos estudios se realizaron en poblaciones de menos de 1000 habitantes (14). En 1996, Chiapas fue escogida como área demostrativa nacional para evaluar esa estrategia. Ese año, la eficiencia del tratamiento llegó a 95\% debido a la supervisión diaria del tratamiento por parte del personal sanitario en las clínicas o en los domicilios de los pacientes. Sin embargo, esto requirió el desvío del personal de otros programas de salud, por lo que no fue posible mantenerlo, y una gran parte del éxito se debió a la voluntad política, la supervisión de los responsables nacionales y estaduales y a la disposición del personal de salud y de los pacientes (20). A pesar de la eficacia del tratamiento acortado estrictamente supervisado, las dificultades existentes en los servicios de salud y el contexto sociocultural de la región plantean la necesidad de evaluar e implantar estrategias combinadas que abarquen la educación sanitaria, con vistas a mejorar a corto plazo el diagnóstico de la tuberculosis y su tratamiento $\mathrm{y}$ obtener un mayor impacto a largo plazo $(15,16)$. Este fue el objetivo del presente estudio realizado en el estado de Chiapas.

En las reuniones de los grupos de autoayuda, los pacientes con tuberculosis mencionaron las dificultades para obtener el diagnóstico y llevar el tratamiento e 
identificaron las barre-ras existentes para la supervisión diaria del tratamiento, en particular las dificultades de transporte y la carencia de recursos económicos para asistir a las unidades de salud. Actualmente se ha ampliado la cobertura de los servicios de salud y, según la estrategia evaluada en este estudio, es posible que pueda capacitarse el personal necesario para atender a los pacientes en sus propias comunidades.

Durante el desarrollo de esta investigación se constataron dificultades en la relación entre el médico y el paciente, por lo que se debe brindar mayor atención al desarrollo de actitudes y aptitudes en el personal médico que conduzcan a un mayor acercamiento a los pacientes y les permita comprender las causas del retraso en el diagnóstico y de que no cumplan el tratamiento los pacientes.

La estrategia educativa mostró una asociación significativa con el cumplimiento del tratamiento indicado. El cumplimiento del tratamiento alcanzado en el grupo de intervención $(97,7 \%)$ es alto para esta región, si se toman en cuenta los antecedentes epidemiológicos y los problemas de pobreza y de marginación predominantes en la mayoría de sus municipios. Sin embargo, no fue posible definir la contribución de cada uno de los componentes de la intervención capacitación médica y grupos de autoayuda- a este resultado, ya que ambas acciones fueron aplicadas en conjunto.

Los materiales utilizados para las reuniones con los pacientes fueron mínimos —principalmente lápices, tarjetas y rotafolios-, por lo que la factibilidad de implantar esta estrategia en otras unidades de salud es elevada. Como parte del proyecto se diseñó un manual dirigido al personal de salud con orientaciones sobre cómo formar los grupos de autoayuda y la información básica sobre la prevención, el diagnóstico y el tratamiento de la tuberculosis, los pasos a seguir en la formación de los grupos de autoayuda y cartas descriptivas de las sesiones.

\section{CONCLUSIONES}

Mediante las actividades educativas emprendidas se logró aumentar significativamente la proporción de pacientes que cumplieron el tratamiento.
Este estudio demostró que los servicios de salud pueden mejorar el control de la tuberculosis en Chiapas con los recursos de los que disponen actualmente. Los resultados indican la necesidad de poner en marcha actividades específicas de refuerzo, entre ellas mejorar los planes de estudio y de servicio social para inculcarles a los médicos una visión integral de los problemas de salud de la región, fomentar una mejor relación entre el médico y el paciente y propiciar que los servicios de salud abran espacios educativos y de participación comunitaria que permitan enfrentar de manera global este problema de salud pública.

Agradecimientos. Este proyecto fue financiado por el Sistema de Investigación Benito Juárez, del Sistema SEP / CONACyT de Oaxaca, México. Se agradece el apoyo del Dr. José Juan Solórzano Moguel, jefe de la Jurisdicción Sanitaria III del Instituto de Salud del Estado de Chiapas, y del personal de las unidades de salud de esa institución.

\section{REFERENCIAS}

1. Fleenor ME, Curtis G. Screening for tuberculosis. JAMA 1995;274(24):1913.

2. Sudre P, Ten Dam G, Kochi A. Tuberculosis: an overview of the situation today. Bull WHO 1991;70(2):149-159.

3. Organización Mundial de la Salud. Grupos en riesgo. Informe sobre la epidemia de tuberculosis, 1996. Ginebra: Programa Mundial contra la Tuberculosis, OMS; 1996.

4. Fauci AS. The AIDS epidemic. Considerations for the 21st century. N Engl J Med 1999; 341(14):1046-1050.

5. México, Secretaría de Salud, Subsecretaría de Prevención y Control de Enfermedades, Programa Nacional de Prevención y Control de la Tuberculosis. Manual de procedimientos. México, D.F.: Secretaría de Salud; 1999

6. México, Sistema Nacional de Información en Salud. Mortalidad en mujeres y hombres (estandarizada por edad) por enfermedades transmisibles, perinatales y deficiencias de la nutrición, según entidad federativa de residencia habitual, 2000. [Sitios en Internet]. http://www.ssa.gob.mx/apps/htdocs/ estadisticas/estadisticas/mortalidad/2000/ Mortalidad_C14_2000.xls y http://www. salud.gob.mx/apps/htdocs/estadisticas/ estadisticas/mortalidad/2000/Mortalidad C15_2000.xls. Acceso el 24 de noviembre de 2003.

7. Instituto de Salud del Estado de Chiapas. Programa de Prevención y Control de la Tuberculosis. Tuxtla Gutiérrez, Chiapas, México: Secretaría de Salud; 1999.

8. Secretaría de Hacienda. Agenda Estadística de Chiapas, 1999. Tuxtla Gutiérrez, Chiapas, México: Secretaría de Hacienda, Dirección de Geografía y Estadística, Gobierno del Estado de Chiapas; 1999.

9. Jaramillo E. Tuberculosis control in less developed countries: can culture explain the whole picture? Tropical Doctor 1998; 28:196-200.

10. Menegoni L. Conceptions of tuberculosis and therapeutic choices in highland Chiapas, Mexico. Med Anthropol Q 1996;10(3):381-401.
11. Álvarez-Gordillo GC, Álvarez-Gordillo JF, Dorantes-Jiménez JE, Halperin-Frisch D. Percepciones y prácticas relacionadas con la tuberculosis y la adherencia al tratamiento en Chiapas, México. Sal Pub Mex 2000;43(1):520528.

12. Álvarez-Gordillo GC, Dorantes-Jiménez JE, Molina-Rosales D. Búsqueda de atención para la tuberculosis en Chiapas, México. Rev Panam Salud Publica 2001;9(5):285-293.

13. Instituto de Salud del Estado de Chiapas, Jurisdicción Sanitaria III. Programa de cómputo EPITB de registro de casos de tuberculosis. Tuxtla Gutiérrez, Chiapas, México: Instituto de Salud del Estado de Chiapas; 2000.

14. Volmink J, Matchada P, Gamer P. Directly observed therapy and treatment adherence. Lancet 2000;355:1345-1349.

15. Jaramillo E. The impact of media-based health education on tuberculosis diagnosis in Cali, Colombia. Health Policy Plan 2001;16(1):68-73.

16. Dick CL. Shared vision-a health education project designed to enhance adherence to 
anti-tuberculosis treatment. Int J Tuberc Lung Dis 1997;2:181-186.

17. Instituto de Salud del Estado de Chiapas, Dirección de Planeación, Información y Evaluación. Catálogo de unidades médicas. Tuxtla Gutiérrez, Chiapas, México: Secretaría de Salud; 1999.

18. Secretaría de Salud. Modificación a la Norma Oficial Mexicana NOM-006-SSA2-1993, para la prevención y control de la tuberculosis en la atención primaria a la salud. Diario Oficial de la Federación (México) 2000, octubre 31.
19. Epi Info version 6.04. Geneva: Centers for Disease Control and Prevention (USA), World Health Organization; 1997.

20. Wayne WD. Pruebas de hipótesis. En: Bioestadística. Base para el análisis de las ciencias de la salud. México, D.F.: Editorial Limusa; 1983. Pp. 155-191.

21. World Health Organization. Global tuberculosis control. Communicable diseases report. Geneva, Switzerland: WHO; 1999.

22. Chaulk PC, Kazandjian VA. Directly observed therapy for treatment completion of pulmonary tuberculosis. JAMA 1998;279:943948.
23. Álvarez-Gordillo GC, Dorantes-Jiménez JE. Tratamiento supervisado para tuberculosis pulmonar en Chiapas. Sal Pub Mex 1998;40: 272-275.

Manuscrito recibido el 17 de marzo de 2003. Aceptado para publicación, tras revisión, el 22 de septiembre de 2003.

ABSTRACT Objective. To implement a training program for physicians and patients and assess its effectiveness in terms of patient compliance with the pulmonary tuberculosis treatment regimen in the border region of Chiapas, Mexico.

Educational strategy for improving patient compliance with the tuberculosis treatment regimen in Chiapas, Mexico
Methods. A controlled intervention study was performed with patients over 15 years of age who had pulmonary tuberculosis diagnosed by direct microscopy (bacilloscopy) between 1 February 2001 and 31 January 2002 in health units randomly selected in the border region of Chiapas, Mexico. The sample was made up of patients who sought consultation at 23 and 25 health units over that period (intervention and control group, respectively). The intervention group took part in a training program for health personnel in which the following were discussed: the social, cultural, and economic aspects of tuberculosis; the theoretical and practical underpinnings of the diagnosis and treatment of the illness, and the establishment of self-help groups. Selfhelp groups were also created for all patients at the 23 units where the intervention group sought consultation. All patients were given a short-term treatment regimen with isoniazid, rifampin, pyrazinamide, and ethambutol for a total of 25 weeks, until completing a total of 105 doses. Patient follow-up was extended through December 2003. The intervention and control groups were compared by means of the chi square test, and Student's $t$ test was used to compare means. The relative risk of noncompliance (RR) was calculated along with $95 \%$ confidence intervals (95\% CI).

Results. Eighty-seven patients participated in the study; 44 were exposed to the intervention, and 43 made up the control group. Compliance with treatment was considerably greater in the intervention group than in the control group $(97.7 \%$ vs. $81.4 \%$, respectively; $\mathrm{RR}=1.20 ; 95 \% \mathrm{CI}: 1.03$ to $1.39 ; P=0.0015)$. It was noted that physicians in the border region of Chiapas gear their activities toward curative medicine, rather than preventive medicine or understanding the social determinants of disease.

Conclusions. As a result of the educational activities that were part of the intervention, there was an increase in the proportion of patients who complied with treatment. Health services can improve tuberculosis control in Chiapas with the resources that are available to them at present. Physicians should be taught to view health problems in Chiapas as part of an integral set of conditions, and efforts should be made to improve the doctor-patient relationship. Steps should also be taken to incorporate educational activities and community participation in health services in order to address public health problems in a comprehensive way. 